\title{
FLUCTUACIÓN POBLACIONAL DE OTOTYLOMYS PHYLLOTIS MERRIAM, 1901 (RODENTIA: MURIDAE) EN UNA SELVA MEDIANA SUBCADUCIFOLIA DEL SUR DE YUCATÁN, MÉXICO
}

\author{
Silvia F. Hernández-Betancourt ${ }^{1}$, José A. Cimé Pool ${ }^{1}$, \\ Salvador Medina Peralta ${ }^{2}$ y Minelia L. González-Villanueva ${ }^{1}$ \\ ${ }^{1}$ Campus de Ciencias Biológicas y Agropecuarias, Universidad Autónoma de Yucatán. \\ Km 15.5 Carretera Mérida-Xmatkuil C.P. 97000 Mérida, Yucatán, MÉXICO \\ hbetanc@uady.mx, cimepool@gmail.com \\ 2Facultad de Matemáticas, Universidad Autónoma de Yucatán. Periférico Norte \\ Tablaje 13615. Apdo. Postal 172 Cordemex, CP 97119. Mérida, Yucatán, MÉXICO. \\ mperalta@uady.mx
}

\section{RESUMEN}

Se estudió la dinámica poblacional de la rata arborícola Ototylomys phyllotis en la selva mediana subcaducifolia del Rancho Hobonil, Tzucacab, Yucatán. Se trabajó de abril de 1996 a abril de 1998, usando el método de captura recaptura y trampeando por cinco noches consecutivas cada mes. La densidad se estimó mediante el método de Número Mínimo de Individuos Vivos (NMIV). Se capturaron un total de 123 individuos, de los cuales el 49\% fueron hembras y el 51\% machos. La proporción de sexos se mantuvo 1:1. La densidad poblacional fluctuó entre 10 y 46 individuos/ha. No se presentó correlación significativa entre la densidad y la precipitación pluvial anual $(\mathrm{p}<0.05)$. Los adultos constituyeron el principal componente de la población. La presencia de reproductores de ambos sexos, así como la actividad reproductiva de las hembras durante la mayoría de los meses de estudio, sugiere que esta especie presenta un patrón reproductivo poliéstrico continuo.

Palabras clave: Poblaciones, Muridae, Ototylomys phyllotis, Selva mediana subcaducifolia, Yucatán, México.

\begin{abstract}
Population dinamics of the big-eared climbing rat (Ototylomys phyllotis) was studied in a subdeciduous tropical rainforest located at Rancho Hobonil, Tzucacab, Yucatan using a mark-recapture technique, from April 1996 to April 1998 during five nights per 24 months. The minimum-numberknown-alive technique (MNKA) was used to estimate population size. We captured 123 rats, 52\% were females and $48 \%$ males. The sex ratio was 1:1 during this study. Density ranged from 10 to 46 ind./ha. And was not significantly correlated with precipitation $(\mathrm{p}>0.05)$. Presence of reproductive individuals from both sexes during the majorities of the studied months and the reproductive activity from females suggest a continuous poliestric pattern for this species.

Key Words: Populations, Muridae, Ototylomys phyllotis, subdeciduous tropical rainforest, Yucatan, Mexico.
\end{abstract}




\section{INTRODUCCIÓN}

Los estudios sobre dinámica poblacional que exhiben algunas poblaciones de pequeños roedores han llamado la atención de naturalistas y ecólogos (Adler 1998, Klinger, 2006, Lima et al. 2001). Los estudios en pequeños roedores tropicales han cobrado gran interés, ya que proporcionan con cierta facilidad información sobre la demografía y reproducción de las poblaciones (Begon 1979, Fleming 1971, 1974a, Kelly \& Caro 2003) que actúan modificando la estructura y función de la selva tropical (DeMattia et al. 2004). Es relevante considerar la existencia de aspectos del hábitat como la variabilidad, estacionalidad, y heterogeneidad de la selva que influyen directamente sobre el patrón demográfico y reproductivo de los organismos (Adler 1998, Fleming 1992). De esta manera las limitaciones biológicas de cada grupo, en conjunto con las características del medio donde habitan, dan como resultado una determinada respuesta de las poblaciones (Caro et al. 2001, Klinger 2006). En México, son varios los estudios sobre dinámica poblacional realizados en bosques templados (Galindo-Leal y Krebs 1997, Santos et al. 2007) y en selvas tropicales (Sánchez-Cordero 1993, Romero 1993, Hernández-Betancourt 2003, Zalapa et al. 2005) en ellos se pueden ver las respuestas de las poblaciones a las variaciones climáticas, estableciendo la estacionalidad de los patrones de fluctuación poblacional de las especies estudiadas.

En el estado de Yucatán la biología de las especies de pequeños roedores es desconocida por lo que, es necesario realizar los estudios pertinentes para determinar los factores ambientales que han modelado su evolución en la selva que habitan y esclarecer los factores bióticos y abióticos implícitos en las estrategias que desarrollan para sobrevivir en este ecosistema y que están relacionadas a sus hábitos alimenticios, ciclos de reproducción y comportamiento.

También es importante establecer las interacciones planta animal que puedan repercutir en la conservación de la selva. Ototylomys phyllotis es una de las 15 especies de pequeños roedores presentes en la Península de Yucatán, presenta hábitos principalmente arborícolas, por lo que los estudios enfocados a ella son limitados (Lawlor 1969, 1982). Resalta también el hecho de que ha sido descrita como reservorio de Leishmania spp. (Chablé et al. 1995) y Trypanosoma sp. (Com pers) y se ha descrito que es parasitada por Cuterebra sp. en el sur de Yucatán (Manrique et al. 2000).

Este estudio es la primera aportación al conocimiento del comportamiento poblacional y aspectos reproductivos de la especie en la selva mediana del sur de Yucatán que se hace con un método sistematizado en campo. 


\section{MATERIALES Y MÉTODOS}

\section{Área de estudio}

El estudio se realizó en el Rancho Hobonil en el municipio de Tzucacab Yucatán, ubicado a $20^{\circ} 00^{\prime} 06^{\prime \prime} \mathrm{N}$ y $89^{\circ} 02^{\prime} 33^{\prime \prime} \mathrm{W}$, al sur del estado. El clima $\mathrm{Aw}_{1}$, (x') $\mathrm{Aw}_{\mathrm{O}}$ ( $\mathrm{x}$ '), es subhúmedo con lluvias en verano y larga temporada de secas (INEGI 1988). El promedio anual de precipitación fluctúa entre 800 y $1200 \mathrm{~mm}$, presentándose las lluvias más abundantes de junio a septiembre (INEGI 1989, Orellana et al. 2003). La temporada de lluvias ocurre de mayo a octubre y la de secas de noviembre a abril, aunque en el sur de Yucatán las se registran algunas lluvias en el período de secas (Duch 1988, Orellana et al. 2003). El promedio de temperatura anual fluctúa entre $22^{\circ} \mathrm{C}$ y $26^{\circ} \mathrm{C}$ (INEGI 1989 ).

La vegetación en el área de estudio está constituida por selva mediana subcaducifolia con elementos de selva mediana subperennifolia (Rzedowski 1987, White \& Hood, 2004). Entre las especies dominantes en la zona de estudio se encuentran: Acacia gaumeri, Randia gaumeri, Bursera simaruba, Croton glabellus Diospyros veraecrucis, D. cuneata, Leucaena leucocephala, Lysiloma latisiliquum, Piscidia piscipula, Lonchocarpus xuul, Sabal yapa, Vitex gaumeri y Licaria peckii entre otras (Flores \& Espejel 1994).

\section{Trabajo de campo}

Este trabajo derivó de un estudio en el cual la especie de interés fue Heteromys gaumeri pero al encontrar a Ototylomys phyllotys como segunda especie más abundante, se decidió analizar la información registrada. El área de estudio comprendió tres cuadrante de $4000 \mathrm{~m}^{2}$ cada uno (12 $000 \mathrm{~m}^{2}$ totales), estos tuvieron las siguientes localizaciones: primero $20^{\circ} 00^{\prime} 55^{\prime \prime} \mathrm{N}$ y $89^{\circ} 02^{\prime} 25^{\prime \prime} \mathrm{W}$, segundo $20^{\circ} 01$ ' $14 " \mathrm{~N}$ y $89^{\circ} 02$ ' $15^{\prime \prime}$ W, y el tercero $20^{\circ} 00^{\prime} 52^{\prime \prime} \mathrm{N}$ y $89^{\circ} 02^{\prime} 19^{\prime \prime}$ W y se colocaron en el mismo tipo de vegetación (selva mediana subcaducifolia).

El establecimiento de tres cuadrantes fue con la finalidad de obtener registros en un área lo mas amplia posible así como una mayor captura de individuos. En cada cuadrante se colocaron 40 trampas Sherman $(8 \times 9 \times 23 \mathrm{~cm})$ en cuatro columnas y 10 filas, con equidistancia de $10 \mathrm{~m}$, constituyendo 120 estaciones de trampeo. Su cebo fueron semillas de girasol. Se empleó el método de captura y recaptura (Krebs 1985), trampeando durante cinco noches consecutivas de cada mes durante 24 meses, de abril de 1996 a abril de 1998. En el último período de lluvias, de mayo a octubre de 1998 se colocaron trampas sobre enredaderas y árboles a alturas que oscilaron entre 0.50 y $2 \mathrm{~m}$, en las estaciones de trampeo de los cuadrantes donde se presentaron mayores frecuencias de captura, con la finalidad de tener registros de los estratos más usados por los animales. En los meses de marzo y abril de 1997, se realizaron recorridos nocturnos cada hora entre las 21:00 P.M. y las 2:00 A.M. revisando cada 
una de las trampas y enfocando los troncos de los árboles y las lianas para observar a los ratones sobre ellos, para determinar el horario de actividad de la especie .

Cada individuo capturado en las estaciones de cualquier cuadrante se consideró como una unidad de información. Los animales fueron marcados por ectomización de falanges (Rudran 1996) y posteriormente liberados en el lugar de captura. Los datos que se registraron para cada individuo fueron los merísticos convencionales (Ramírez Pulido et al. 1989), además de: número de marca, ubicación de la trampa, peso, sexo y condición reproductiva. Se consideraron tres categorías de edad joven, subadulto y adulto, de acuerdo a las características morfológicas de los individuos. Al analizar los pesos de los animales y relacionarlos con las características, las edades se determinaron como sigue: Los jóvenes presentaron un promedio de peso de $27.72 \mathrm{~g}$ (d.e. 7.55), órganos sexuales poco desarrollados, en los machos los testículos fueron pequeños y el pelaje fue nuevo y completo. En los subadultos el promedio de peso de fue de $47.3 \mathrm{~g}$ (d.e. 4.30), presentaron testículos escrotados medianos sin sacos epididimales, y el pelaje fue completo de color gris claro a gris oscuro. Para los adultos el promedio de peso de fue de 61.37 (d.e. 8.87), estos presentaron testículos y sacos epididimales bien desarrollados y su pelaje fue completo pero la coloración menos brillante. Las hembras jóvenes pesaron en promedio $32.48 \mathrm{~g}$ (d.e. 4.23), presentaron pezones poco desarrollados y vagina cerrada. En las subadultas el peso promedio de fue de $43.46 \mathrm{~g}$ (d. e. 4.67), las glándulas mamarias estuvieron más desarrolladas y la vagina cerrada. Las adulas no reproductivas, tuvieron peso promedio de $59.07 \mathrm{~g}$ (d.e. 7.62) presentaron glándulas mamarias desarrolladas y la vagina estuvo cerrada. No se consideraron las gestantes. Las reproductivas se consideraron lactantes cuando las glándulas mamarias produjeron leche, postlactantes, cuando los pezones estuvieron grandes y secos, gestantes cuando se pudieron palpar los embriones por medio del tacto y receptivas cuando presentaron la vagina abierta. El pelaje para las tres edades fue similar al de los machos.

El trampeo se calculó considerando el porcentaje de individuos marcados del total de la muestra capturada por mes. También se calculó la eficiencia de captura (número de individuos capturados en total cada mes/total de noches trampa) y la media del número de capturas por individuo considerando el número total de recapturas entre el número total de individuos capturados por mes (Jonsson et al. 2000).

La densidad de la población se calculó usando método de enumeración directa o Número Mínimo de Individuos Vivos (NMIV) (Krebs 1989, Nichols \& Pollock 1983), considerando el área total muestreada $\left(12000 \mathrm{~m}^{2}\right)$ que fue relacionada a una hectárea. El análisis de reclutamiento mensual se realizó con los individuos no marcados de cualquier edad que ingresaron a la población marcada, esto se hizo con la finalidad de apreciar las fluctuaciones reales de la población por medio de esa muestra (Galindo-Leal \& Krebs 1997). Se consideraron individuos residentes aquellos que se capturaron por lo menos en tres períodos de captura. 


\section{Análisis estadístico}

Se empleó la prueba de Wilcoxon de rangos para muestras relacionadas (Wackerly et al. 2002) para determinar si el número de individuos por hectárea calculado por NMIV, fue mayor que los valores registrados en campo. Se aplicó la prueba U de Mann-Withney (Zar 1999) para determinar diferencias en la permanencia entre machos y hembras. Para evaluar la correlación entre la densidad y la precipitación pluvial, que suele presentarse en especies de pequeños roedores en selvas tropicales (Adler 1998, Fleming 1992) se utilizó el coeficiente de correlación por rangos de Spearman (Wackerly et al. 2002). El análisis de varianza se utilizó en un diseño bifactorial para determinar efectos significativos de los factores, edad y época en la raíz cuadrada de la variable captura (Montgomery 1997), con la finalidad de ver si alguna edad de la población se capturaba más durante alguna de las dos épocas. Para las diferencias en la proporción de sexos se usó la prueba ji-cuadrada de homogeneidad (Zar 1999). En todos los casos se usó $\alpha=0.05$.

\section{RESULTADOS}

La comunidad de pequeños roedores estuvo constituida por las siguientes especies: Heteromys gaumeri (85\%), Ototylomys phyllotis (14\%), Peromyscus yucatanicus $(0.6 \%)$, Oryzomys melanotis $(0.3 \%)$, Reithrodontomys gracilis $(0.05 \%)$ y Sigmodon hispidus $(0.05 \%)$. La rata $O$. phyllotis fue la segunda especie más abundante. Se marcaron un total de 123 individuos con 356 recapturas, con un esfuerzo de captura de 14400 noches trampa. El 48\% de la población total fue de hembras y el $52 \%$ de machos. La proporción de sexos de la población total se mantuvo 1:1 $\left(X^{2}=0.012\right.$, g.l. $\left.=1, P=0.914\right)$ durante todo el estudio. Los machos tuvieron un promedio de permanencia de 7.5 meses (d.e. 4.57) y sólo uno permaneció por 21 meses, mientras que las hembras permanecieron 7.04 meses (d.e. 3.22) con un máximo de 13 meses. No hubo diferencia entre la permanencia de machos y hembras $(U=290, P=0.9752)$.

\section{Trampeo y capturabilidad}

La captura total de individuos registró un incremento de mayo a noviembre de 1997 y de diciembre a abril bajó ligeramente. El promedio de individuos totales capturados fue de 15.13 (d.e. 7.03) y el de recapturas fue de 12.13 (d.e. 9.80). Las mayores capturas ocurrieron en septiembre y octubre de 1996, y de julio a septiembre y noviembre de 1997. Las capturas mínimas se presentaron durante junio de 1996 y abril de 1997 (Fig 2). Las recapturas durante el estudio constituyeron un 50\% o más de la captura total. No se presentaron recapturas en mayo de 1996 y en diciembre de 1997. El promedio de eficiencia de captura mensual fue de 0.024 (d.e. 0.010$) \mathrm{y}$ 
fluctuó entre 0.008 y 0.046 , lo cual indica que la población en general es muy baja. La media de captura mensual por individuo fue de 1.7 (d. e. 0.4037) y fluctuó entre 1 y 2.45 capturas/individuo. La prueba de ANOVA no determinó un efecto significativo de interacción entre edad y época $(F=1.85, P=0.1803)$, tampoco en la época $(F=0.85, P=0.3624)$, pero sí de la edad $(F=36.96, P<0.0001)$, es decir los adultos fueron capturados más que los jóvenes, sin importar la época.

\section{Tamaño de la población}

La densidad poblacional de $O$. phyllotis de acuerdo con los valores registrados, fluctuó entre 6 y $36 \mathrm{ind} / \mathrm{ha}$ y aplicando el NMIV entre 10 y 46 ind/ha. Se presentaron diferencias significativas entre los valores registrados durante el estudio y los calculados por el método $(T=0.0, P<0.0001)$ el cual sobrestimó la población. Durante el tiempo de estudio se observó una tendencia al incremento continuo de la densidad, sin embargo, los picos máximos por año se presentaron entre los meses de julio y diciembre de 1996 (18 a 27 ind/ha) y de julio a diciembre de 1997 (32 a 46 $\mathrm{ind} / \mathrm{ha}$ ). No hubo correlación significativa entre las densidades poblacionales y la precipitación pluvial mensual $\left(r_{s}=0.1797, P=0.3888\right)$ (Fig. 1).

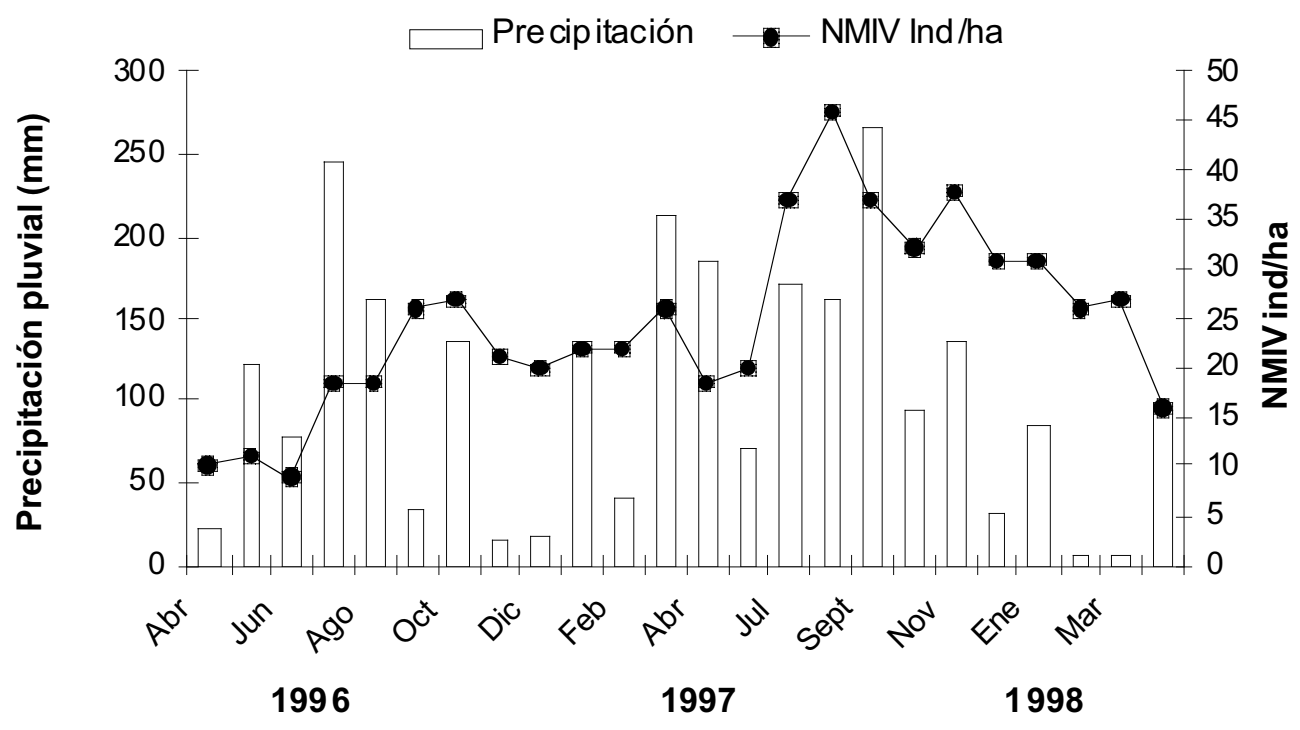

Figura 1. Precipitación pluvial y densidad poblacional de Ototylomys phyllotis en una selva del sur de Yucatán. 


\section{Reclutamiento}

El mayor reclutamiento para las tres edades se observó durante julio de 1997 (18 ind.) y el menor en diciembre de 1996 (1 ind.). En abril de 1997 y febrero de 1998 no hubo entradas de nuevos individuos. El reclutamiento total estuvo constituido por 22 machos y 32 hembras adultos, 24 machos y 12 hembras subadultos, y 19 machos y 14 hembras jóvenes. La entrada de reclutas jóvenes no presentó un patrón definido, hubo fluctuaciones de 1 a 2 individuos de abril de 1996 a abril de 1997 y un pico máximo en julio de 1997 (8 ind.). Los subadultos presentaron fluctuaciones con entradas de hasta cuatro individuos durante la época de lluvia de 1996 y 1997, y de sólo un individuo en la época de secas de 1997. Los adultos, al igual que los jóvenes, no presentaron un patrón definido, hubo entradas de 1 a 4 individuos de abril de 1996 a abril de 1997 y la máxima se registró en julio de 1997 (7 ind) (Fig. 2).
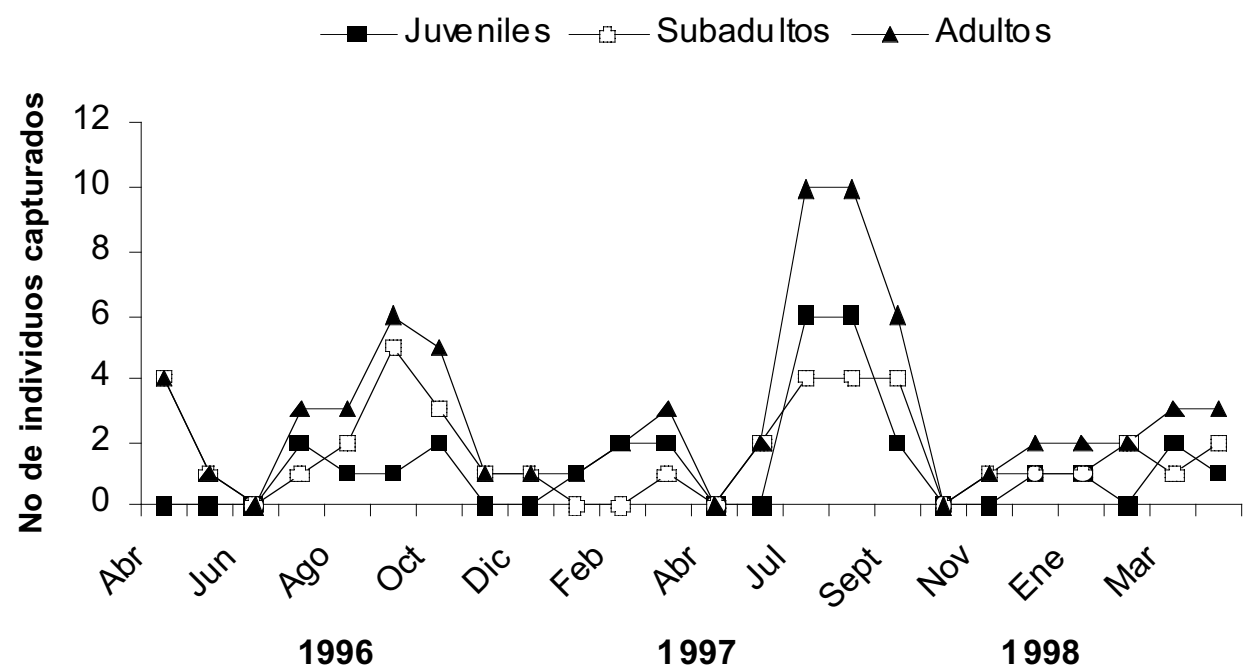

Figura 2. Reclutamiento mensual por edades de Ototylomys phyllotis en una selva del sur de Yucatán.

\section{Estructura por edades}

El mayor componente de la población considerando las recapturas $(n=356)$ lo constituyeron los adultos (74\%), seguidos de los subadultos $15 \%$ y el menor componente fueron los jóvenes $11 \%$. El pico de los adultos se observó durante noviembre de 1997 (21 ind.) y el menor valor en abril de 1996 (3 ind.). El mayor número de subadultos ( 7 ind.) se presentó en agosto de 1997 y no se presentaron en junio de 1996. Los jóvenes presentaron su máximo (8 ind.) en agosto de 1997, y no se registraron durante seis meses (Fig. 3). 

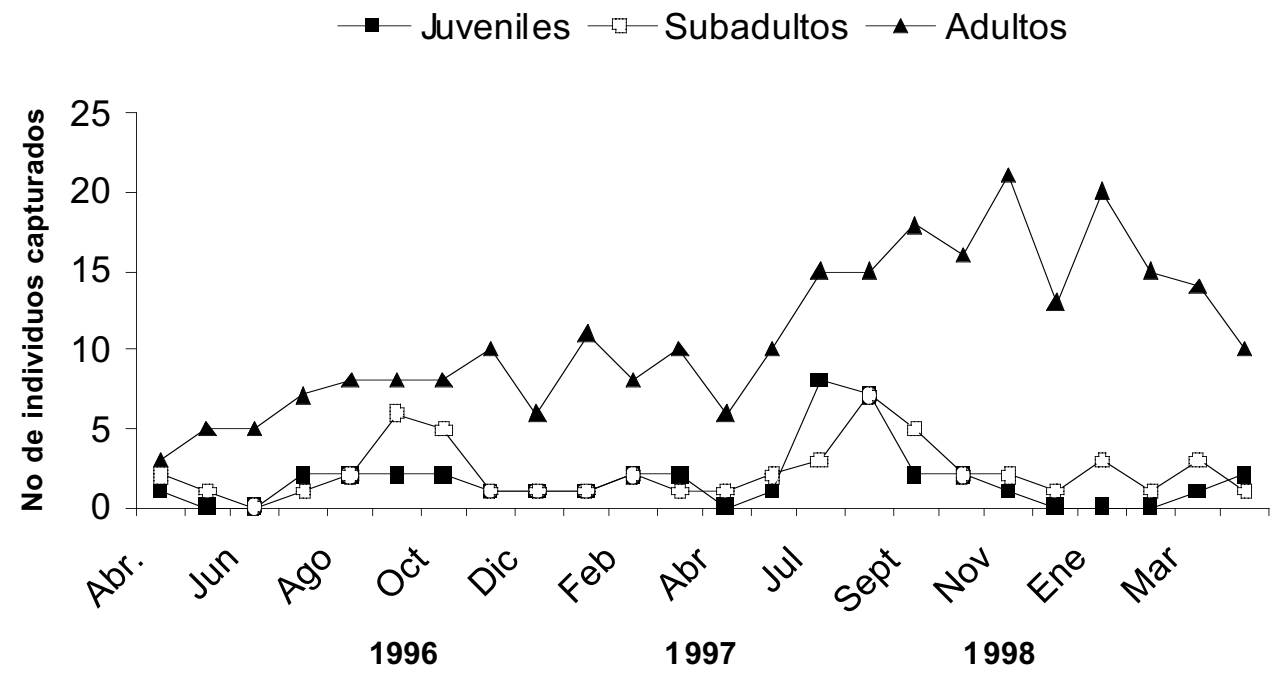

Figura 3. Estructura por edades de Ototylomys phyllotis en una selva del sur de Yucatán.

El análisis de edades por sexo siguió el mismo patrón que la estructura por edades total. El máximo de machos adultos (13 ind.) se presentó en septiembre de 1997 y el de las hembras en enero de 1998 (11 ind.). En abril y mayo de 1996 no se capturaron machos, en contraste, las hembras se capturaron durante todo el estudio, con fluctuaciones de dos a 11 individuos.

\section{Patrón Reproductivo}

La población total de individuos reproductivos durante los dos años fue de 54, de los cuales, el $40 \%(n=22)$ correspondió a machos y el $60 \%(n=32)$ a hembras. Considerando las recapturas en todos los meses se capturaron hembras reproductivas (Cuadro 1). La proporción de sexos de los individuos reproductivos se mantuvo 1:1 durante el tiempo de estudio $\left(X^{2}=2.46, g . l .=1, P>0.05\right)$. Las hembras con actividad reproductiva $(81 \%)$, se presentaron durante todos los meses. Durante 10 meses el $100 \%$ de hembras registradas presentaron alguna actividad reproductiva. Las hembras adultas no reproductivas $(\mathrm{n}=23)$ se presentaron durante 14 meses con pocos individuos (1 a 3) (Cuadro 1). Entre las hembras reproductivas ( $n=109)$, las receptivas $(\mathrm{n}=34)$ se registraron durante 17 meses presentándose en números bajos $(1$ a 3 ind.). Las hembras gestantes se observaron durante 16 meses también en números bajos (1 a 3 ind.) con un máximo (5 ind.) en marzo de 1998 (Cuadro 1). Las lactantes se presentaron también durante 15 meses, fluctuando entre uno y tres individuos y las postlactantes se registraron durante 14 meses, de abril de 1996 a abril de 1997 se presentaron en números bajos (1 a 2 ind.), pero durante la época seca de 1998 presentaron su máximo (4 ind). 
Cuadro 1. Estado reproductivo de hembras de Ototylomys phyllotis en una selva del sur de Yucatán de abril de 1996 a abril de 1998.

\begin{tabular}{|c|c|c|c|c|c|c|}
\hline & Receptivas & Gestantes & Lactantes & Postlactantes & No Reproductivas & $\%$ Rep \\
\hline$\overline{\mathrm{Abr}}$ & 2 & 0 & 1 & 0 & 0 & 100 \\
\hline May & 1 & 1 & 2 & 0 & 1 & 80 \\
\hline Jun & 1 & 0 & 1 & 1 & 0 & 100 \\
\hline Jul & 2 & 1 & 1 & 2 & 1 & 86 \\
\hline Ago & 0 & 1 & 3 & 0 & 0 & 100 \\
\hline Sept & 0 & 3 & 0 & 0 & 0 & 100 \\
\hline Oct & 3 & 0 & 2 & 0 & 0 & 100 \\
\hline Nov & 0 & 1 & 3 & 1 & 0 & 100 \\
\hline Dic & 0 & 0 & 1 & 0 & 1 & 50 \\
\hline Ene & 2 & 1 & 0 & 1 & 0 & 100 \\
\hline Feb & 3 & 1 & 1 & 1 & 1 & 86 \\
\hline Mar & 3 & 1 & 2 & 0 & 1 & 86 \\
\hline Abr & 0 & 0 & 1 & 1 & 3 & 40 \\
\hline May & 0 & 3 & 0 & 2 & 1 & 83 \\
\hline Jul & 2 & 3 & 1 & 1 & 1 & 87 \\
\hline Ago & 1 & 0 & 1 & 1 & 3 & 50 \\
\hline Sept & 2 & 0 & 0 & 1 & 4 & 43 \\
\hline Oct & 3 & 1 & 1 & 0 & 2 & 75 \\
\hline Nov & 2 & 1 & 1 & 4 & 0 & 100 \\
\hline Dic & 1 & 2 & 0 & 0 & 1 & 75 \\
\hline Ene & 3 & 3 & 0 & 2 & 1 & 89 \\
\hline Feb & 2 & 1 & 0 & 4 & 0 & 100 \\
\hline Mar & 1 & 5 & 0 & 0 & 0 & 100 \\
\hline Abr & 0 & 0 & 0 & 2 & 2 & 50 \\
\hline
\end{tabular}

El seguimiento de cinco hembras marcadas permitió ver que la gestación probablemente dura de 30 a 45 días, ya que el intervalo a partir de que se capturaron con la vulva abierta hasta la lactancia varió entre uno y dos meses. La lactancia probablemente dura 30 días, ya que nueve hembras lactantes al siguiente mes se registraron como postlactantes. El seguimiento de una hembra durante 13 meses permitió observar tres gestaciones, con una diferencia de seis meses entre la primera a la segunda (intercalados dos meses de receptividad) y un mes (postlactancia) entre la segunda a la tercera captura. Tres hembras presentaron dos gestaciones, dos de ellas con intervalo de un mes y la otra de cuatro meses. Veintidós hembras 
presentaron solo una gestación. Se observaron dos hembras que parieron en las trampas, tres crías cada una, estas estaban sujetas a los pezones, su peso promedio fue de $2.75 \mathrm{~g} \mathrm{(d.e.} 0.274)$. El seguimiento de 16 hembras preñadas permitió observar que en promedio pierden $13 \mathrm{~g}($ d. e. 6.49) después de parir a las crías, siendo este el $17 \%$ (d.e. 7.78$)$ del peso corporal el promedio del peso de la camada.

\section{Actividad arborícola y nocturna}

Del total de la población $(\mathrm{n}=123)$, el $24 \%(\mathrm{n}=30)$ de los individuos, todos adultos, se capturaron sobre árboles siendo el $60 \%$ machos y el $40 \%$ hembras. Las alturas fluctuaron entre 0.50 y $2 \mathrm{~m}$, siendo la más frecuente la de $1.50 \mathrm{~m}$. Al liberar a los animales se logró observar a tres individuos trepar por los bejucos, uno de ellos hasta $3 \mathrm{~m}$, para posteriormente bajar a su madriguera. El mayor registro de actividad nocturna de $O$. phyllotis se observó entre las 23:00 P.M. y la 1:00 A.M durante este mismo tiempo se capturaron principalmente en las trampas sobre el suelo y se observaron al alumbrar sobre troncos de bejucos y en una ocasión sobre el árbol de dzidzilché Gymnopodium floribundum.

\section{DISCUSIÓN}

La eficiencia de captura y la media de captura por individuos reflejan que la población de Ototylomys phyllotis es baja, comparada con la de Heteromys gaumeri (Hernández-Betancourt 2003, Rasmussen-Terán 2006), los porcentajes de captura durante el estudio fueron de $15 \%$ y $83 \%$ respectivamente. Lo que coloca a $O$. phyllotis lo que la coloca como la segunda especie en abundancia en la zona de estudio probablemente debido a su continua actividad reproductiva (Cuadro 1) que mantiene a la población, cuyo incremento podría estar limitado por la depredación de aves como Tyto alba, Glaucidium brasilianum y mamíferos como Urocyon cinereoargenteus, Procyon lotor y Leopardus wiedii que fueron observados en la zona de estudio y considerando que durante los recorridos nocturnos se vio a $O$. phyllotis sobre lianas y árboles. En Las Cuevas, Belice se observó que la presencia de depredadores y las condiciones del suelo fueron factores que causaron bajas densidades de pequeños roedores $(2.7 / \mathrm{ha})$ entre los que se encontró $O$. phyllotis (Kelly \& Caro 2003), así mismo se ha documentado la depredación diferencial por dos especies de aves nocturnas sobre poblaciones de Phyllotis darwini en la vegetación de climas semiáridos en Chile (Farias \& Jacksic 2007, Lima et al. 2001).

En otros estudios sobre pequeños roedores realizados en selvas de Belice se señala a $O$. phyllotis como especie relevante, en la selva baja en la Reserva de Bladen, esta especie estuvo entre las tres más capturadas (Klinger 2006). En 
contraste, en la selva húmeda de la Reserva Shipstern, fue la más abundante con el 90.8\% de las capturas totales (Bersot 2003).

El hecho de que la densidad poblacional de $O$. phyllotis se mantuviera durante todo el período de estudio, probablemente se deba a la disponibilidad de alimento de la selva en la que se realizó el estudio, ya que en el sitio la precipitación ocurre durante todo el año aunque se intensifica de de julio a septiembre (Fig. 1), y por lo menos hay 36 especies de plantas fructifican de 3 a 12 meses (Hernández-Betancourt et al. 2003). La productividad relacionada a la permanencia de las densidades poblacionales ha sido observada para otras especies como Prochimys semispinosus en algunas islas de Panamá, ya que el aumento o disminución de esta especie depende de la producción de frutos producidos (Adler, 1998, Adler \& Lambert, 1997). En O. phyllotis una característica intrínseca es su capacidad de forrajeo tanto en el piso como sobre los árboles en los que se alimenta de hojas, frutos y semillas (Lawlor 1982), lo cual ayuda a que la población se mantenga. Durante la época seca se observó alimentándose de corteza de troncos. La abundancia de alimento en la zona de estudio también se vio reflejada en la población de $H$. gaumeri cuyas poblacionales mantuvieron sus densidades durante 24 meses (Hernández-Betancourt et al. 2003). La densidad poblacional de 10 a 46 ind/ha obtenida para $O$. phyllotis en este estudio es menor a la observada por los autores en una selva baja caducifolia conservada de la Reserva Estatal de Dzilam, Yucatán en donde fue la primera especie en abundancia con densidades entre 4 y $64 \mathrm{ind} / \mathrm{ha}$ (Cimé 2006). La densidad de $O$. phyllotis es similar a la de otras especies de múridos como la registrada para Peromyscus aztecus ( 8.5 a más de $30 \mathrm{ind} / \mathrm{ha}$ ) en la selva mediana subcaducifolia del oeste de Jalisco, México (Vázquez et al. 2000).

Los cambios en la dinámica poblacional de especies de pequeños roedores en relación con la estacionalidad en la selva tropical ha sido discutida (Adler 1998, Fleming 1992) señalando que la época de lluvias coincide con el aumento poblacional debido a la gran productividad de la época. Para $O$. phyllotis la densidad poblacional no tuvo correlación con la precipitación considerando todo el período de estudio, sin embargo de julio a noviembre de 1997 se presentaron las mayores densidades y precipitaciones (Fig. 1) lo cual podría indicar que la productividad favorece el incremento de la poblacional de esta especie, aunque esto se comprobaría incrementando los períodos de muestreo por lo menos a 5 años (Crespin \& Lima 2006). Se han observado patrones de correlación positiva en especies como $P$. semispinosus (Adler, 1998) Heteromys desmarestianus (Sánchez-Cordero, 1993) Phyllotis darwini (Lima et al. 2001).

La dinámica de reclutamiento de $O$. phyllotis parece indicar que en el período de estudio se presentaron dos ciclos, ya que de agoto a noviembre de 1996 se presentó un máximo que incluyó las tres edades, lo mismo se observó de agosto a octubre de 1997 (Fig. 2). Los adultos fueron capturados durante la mayoría de los meses sin 
importar la época, como lo demostró la prueba de ANOVA, esto trae como consecuencia la entrada de jóvenes y subadultos que se enfatizó en la época de lluvias de 1996 y 1997, probablemente por la gran producción de frutos y hojas durante esta época, que les proporciona toda la energía necesaria para el proceso reproductivo. La presencia de adultos reproductivos se ha señalado como fundamental para mantener la sobrevivencia de las poblaciones, de Phyllotis darwini en Chile (Crespin \& Lima 2006). Existen comportamientos particulares a cada sexo que muchas veces ocasionan su muerte o emigración influyendo en la dinámica del reclutamiento. Por ejemplo, en machos, la disminución o ausencia de individuos adultos quizá se deba a las presiones por encontrar y fecundar un mayor número de hembras para preservar sus genes. En contraste, las hembras se ven más interesadas en la búsqueda de alimento y refugios para garantizar el desarrollo de las crías (Bond \& Wolf 1999, Jonsson et al. 2000, Kitchen et al. 2000). Esto se documentó en el área de estudio para Heteromys gaumeri (Hernández-Betancourt et al. 2003).

Durante el estudio fue mayor presencia de adultos y subadultos en la población, durante 1997 se incrementó la presencia de adultos, probablemente debido a la aportación por sucesión de edades y por la inmigración de individuos de otras poblaciones (Figs. 2 y 3). La baja presencia de jóvenes, quizá esté ligada a los hábitos de crianza de $O$. phyllotys, ya que las hembras presentan cuatro pezones y conservan a las crías amamantándolas por lo menos 30 días, lo cual puede reflejarse en un abandono tardío de la madriguera. Además de que los neonatos tienen un peso alto al nacer, así como la poca competencia al amamantarse (generalmente se presentan dos crías), explica que haya un período corto entre las edades y un rápido arribo a la edad adulta y a la reproducción, como lo indicaron Lawlor (1982) y Disney (1968) al trabajar con la especie en condiciones de cautiverio. Esta estrategia reproductiva tal vez sea una respuesta a la competencia interespecífica y la depredación, ya que al destetar a las crías en estado avanzado de desarrollo e iniciar la reproducción en períodos cortos puede ser posible que las densidades de población se mantengan constantes.

Las hembras y machos reproductivos se registraron durante todos los meses de estudio, lo cual sugiere que la reproducción es continua similar al observado por los autores en la selva baja caducifolia de la Reserva Estatal de Dzilam. Una de las estrategias reproductivas conocidas para las hembras de O. phyllotis es ser poliéstricas, por lo que se pueden reproducir durante todo el año (Birney et al. 1974, Lawlor 1969) y presentar celo estral postparto después de 24 horas aproximadamente (Flowerdew 1987, Helm 1975). En este estudio se observaron tres hembras que al mes de ser capturadas como lactantes ya estaban preñadas nuevamente. Lawlor (1982), registró el primer apareo de esta especie a una edad de 29 días en hembras y 175 días en machos. La precocidad reproductiva de las hembras probablemente ayuda a que esta especie sea la segunda más abundante en la zona de estudio. 
En trabajos experimentales no publicados realizados en el Campus de Ciencias Biológicas y Agropecuarias de la Universidad Autónoma de Yucatán, se ha observado que en las hembras la vulva se abre por primera vez en edades que fluctúan entre 21 y 70 días y en los machos los testículos descienden entre los 15 y 38 días de edad. Para la misma especie en la selva tropical de Belice se reportó que ambos sexos llegaron a la madurez sexual a los 39 días (Disney 1968). En los tres casos se observa que la madurez sexual se alcanza a edades tempranas.

La presencia de hembras lactantes y postlactantes durante todo el período de estudio sugiere que los eventos reproductivos tuvieron éxito y que las hembras pudieron cubrir sus requerimientos energéticos adecuadamente en el hábitat en que se desarrollaron. En este estudio se registraron hembras preñadas durante 15 meses. El $85 \%$ de las hembras preñadas solo presentaron una gestación y solo cuatro tuvieron más, esto probablemente se debe a la gran inversión de energía usada para asegurar el desarrollo de las crías, lo cual produce un gran desgaste. En general se ha observado que la cantidad de energía requerida para la producción de crías se incrementa constantemente desde los inicios de la lactancia. Una hembra en etapa tardía de lactancia necesita consumir alrededor de cuatro a cinco veces más alimento que antes de quedar preñada (Stebbins 1977). En contraste los machos invierten la energía disponible en maximizar el acceso a las hembras (Morse 1980, Ostfeld 1985). Quizá esto mantenga activos reproductivamente a los machos de O. phyllotis durante todo el año. En este estudio se registró que 16 animales principalmente machos se encontraban parasitados por Cuterebra predominante mente en la zona de los genitales y algunos individuos presentaron reinfección (Manrique et al. 2000).

En la zona de estudio $O$. phyllotis desarrolla alguna actividad principalmente sobre bejucos horizontales a diferentes alturas, siendo la más frecuentemente la de $1.5 \mathrm{~m}$, aunque se observó a un animal a $3 \mathrm{~m}$ de altura. En la selva baja caducifolia de la Reserva Estatal de Dzilam, Yucatán la altura de mayor actividad fue a $1.6 \mathrm{~m}$ (Cimé 2006). En Costa Rica se reportó que $O$. phyllotis utiliza la vegetación a una altura promedio de 2.3 (1.0 a 3.5 m) (Sáenz 1999). Lawlor (1982) reportó que esta especie utiliza el estrato arbóreo a una altura inferior a los $4 \mathrm{~m}$, lo cual coincide con lo observado en este estudio, aunque el mismo autor no niega que la especie tenga actividad en el suelo.

En el sitio de estudio se observó que $O$. phyllotis se desplaza principalmente en el suelo, debido a que sus madrigueras se encuentran ocultas bajo la superficie y probablemente las comparte con $H$. gaumeri porque al liberar a ambas especies en sus sitios frecuentemente entraban a los mismos agujeros. En una selva baja caducifolia de la Reserva de Dzilam se observó que $O$. phyllotis prefiere sitios con alta pedregosidad (Cimé 2006) lo mismo ha sido visto por los autores en el Parque Nacional de Dzibilchaltún y la Reserva Estatal Lagunas de Yalahau en Yucatán. En este estudio no se observó actividad natural de O. phyllotis durante el día. Sin 
embargo en Costa Rica haciendo seguimiento de animales marcados con microchips, se registró actividad diurna, como en algunas otras especies de múridos neotropicales. Con el mismo método se registró actividad de las 18:00 a las 23:00 hs (Sáenz 1999), aunque Lawlor (1982) la describe como una especie nocturna. En la selva del sur de Yucatán la actividad arborícola de esta especie se registró durante la noche, entre las 23:00 P. M. y 1:00 A.M. aunque se hicieron observaciones a partir de las 21:00 h.

\section{AGRADECIMIENTOS}

Agradecemos a la Dra. Celia López González del CIIDIR-Durango la revisión de este manuscrito, a los Biólogos Candita Euan y Ermilo López por su valiosa colaboración en el trabajo de campo. Por el apoyo logístico en la FMVZ al Sr. Julio Pool y en el Rancho Hobonil, al M. en C. José Erales Villamil, Doña Rosita y Don Catín. La Beca PROMEPUADY-64 y la FMVZ apoyaron financieramente esta investigación.

\section{LITERATURA CITADA}

Adler, G. H. 1998. Impacts of resource abundance on populatons of tropical forest rodent. Ecology. 79(1):242-254.

Adler \& Lambert, 1997. Ecological correlates of trap response of a Neotropical forest rodent, Prochiemys semispinosus. J. Trop. Ecol. 12:59-68.

Begon, B. 1979. Investigating animal abundance: capture-recapture for biologist. Edward Arnold. Great Britain. 97 p.

Bersot, V. 2003. Small mammals inventory in the Shipstern Nature Reserve (Corozal District, Belize, Central America), a preliminary assessment. Rev. Suisse Zool. 110:207-246.

Birney, C. E., J. B. Bowles, R .M. Timm \& S. L. Williams. 1974. Mammalian distributional records in Yucatan and Quintana Roo, with comments on reproduction, structure and status of peninsular population. Occas. Pap. Bul. Museum of Natural History University of Minnesota. No 13.

Bond, M. L. \& J. O. Wolff. 1999. Does access to female or competition among males limit male home-range size in a promiscuous rodent? J. Mamm. 80(4): 1243-1250.

Caro, T.M. , M. J. Kelly, N. Bol \& S. Matola. 2001. Inventorying mammals at the multiples sites inthe maya mountains of Belice. J. of Mamm. 82(1): 43-50.

Chablé, J., N. Van Wynsberghe, S. Canto-Lara y F. Andrade. 1995. Islation of de Leismania(l.) mexicana from wild rodents and their possible role on the transmission of localized cutaneus leishmaniasis in the State of Campeche, México. Amer. J. of Trop. Med. Hyg., 53(2): 141-145.

Cimé Pool, J. A. 2006. Ecología de comunidades de pequeños roedores en un gradiente de perturbación de selva baja caducifolia espinosa de la Reserva Estatal de Dzilam, Yucatán, México. Tesis de Maestría. Campus de Ciencias Biológicas y Agropecuarias. Universidad Autónoma de Yucatán. 64 p. 
Crespin, L \& M. Lima. 2006. Supervivencia adulta y dinámica poblacional del lauchon orejudo Phyllotis darwini en Chile central. Rev. Chilena Hist. Nat. 79:295-308.

DeMattia, E. A., Curran, L. M. \& Rathcke, B.J. 2004. Effects of small rodents and large mammals on Neotropical seeds. Ecology. 85:2161-2170.

Disney, R. H. 1968. Observations on a zoonosis: Leishmaniasis in British Honduras. Applied Ecology, 5:1-59.

Duch, G. J. 1988. La conformación territorial del Estado de Yucatán. Los componentes del medio físico. Universidad Autónoma de Chapingo. Centro Regional de la Península de Yucatán.

Farias, A. y F. M. Jacksic. 2007. Effects of functional constraints and opportunism on the functional estructure of vertebrate predator assemblage. J. of Anim. Ecol. 76: 246-257.

Fleming, T. H. 1971. Population ecology of three species of Neotropical rodents. Miscellaneus Publication. Museum of Zoology, Univ. Michigan, 143:1-47.

. 1974. The population ecology of two species of Costa Rican heteromyid rodents. Ecology, 55:493-510.

. 1992. How do fruit-and nectar-feeding bird and mammals, track their food resources? Pp. 355-391. In: Effects of resources distribution on animal-plant interactions. Academic Press. London.

Flores, J. S. e I. Espejel. 1994. Vegetación de la Península de Yucatán. Etnoflora Yucatanense. Fascículo 3. Universidad Autónoma de Yucatán. Mérida, Yucatán. 135 pp.

Flowerdew, J. R. 1987. Mammals: Their reproductive biology and population ecology. Ed. Edward Arnold. Great Britain. 241 pp.

Galindo-Leal, C. \& C. Krebs. 1997. Habitat structure and demography variability of habitat specialist: The rock mouse (Peromyscus difficilis). Rev. Mex. Mast., 2:72-89.

Helm, J. D. 1975. Biology reproductive of Ototylomys phyllotis (Cricetidae) J. of Mamm. 56(3).

Hernández-Betancourt, S. 2003. Dinámica poblacional de Heteromys gaumeri Allen y Chapman 1897en una selva mediana del sur de Yucatán. Tesis de doctorado. UAM-Iztapalapa. México, D.F. 272 p.

Hernández-Betancourt, S.F., R. López-Wilchis, J. A. Cimé Pool \& S. Medina Peralta. 2003. Área de actividad, movimiento y organización social de Heteromys gaumeri Allen y Chapman, 1897 (Rodentia:Heteromyidae) en una selva mediana subcaducifolia de Yucatán, México. Act. Zool. Mex. (n. s.) 90: 77-91.

INEGI. 1988. Carta de vegetación y uso del suelo. Escala 1:1,000,000. Instituto Nacional de Estadística, Geografía e Informática. México.

INEGI: 1989. Carta climática. Escala 1:1,000,000. Instituto Nacional de Estadística, Geografía e Informática. México.

Jonsson, P., E. Koskela \& T. Mappes. 2000. Does risk of predator affect the spacing behaviour of rodents?. Two large-scale experiments. Oecologia, 122:487-492.

Kelly M.J. \& T. Caro. 2003 Low density of small mammal community at Las Cuevas, Belice. Mammalian Biology. 68: 372-386.

Kitchen, A. M., E. M. Gese \& E. R. Schauster. 2000. Long term spatial of coyote (Canis latrans) home ranges in southeastern Colorado. Can. J. of Zool., 78:458-464. 
Klinger, R. 2006. The interaction of disturbances and small mammal community dynamics in a lowland forest in Belize. J. of Anim. Ecol. 75:1227-1238.

Krebs, C. J. 1985. Ecología. Estudio de la distribución y la abundancia. $2^{\text {a }}$ Edición. Harla. México, D. F. 753 pp. $654 \mathrm{p}$.

Lawlor, T. E. 1969. A systematic study of the rodent genus Ototylomys. J. of Mamm., 50:2842. 1982. Ototylomys phyllotis. Mammalian Species. No. 181:1-3.

Lima, M., R. Julliard, N. C. Stenseth \& F. M. Jaksic. 2001. Demographic Dynamics of a neotropical small rodent (Phyllotis darwini): feedback structure, predation and climatic factors. Ecology.70:761-775.

Manrique-Saide P., S. Hernández B. Y M. T. Quintero. 2000. First record of Cuterebra sp. (Diptera Cuterebridae) infection in Ototylomys phyllotis (Rodentia: Muridae). Florida Entomologist. 83 (4) 487-488.

Montgomery, D. C. 1997. Desing and analises of experiments. $4^{\text {a }}$ Ediciión. John Willey \& Sons. Arizona State University. 341 p.

Morse, D. H. 1980. Behavioral mechanisms in ecology. Harvard Univ. Press. Cambridge, Mass. 383 pp.

Nichols, J. D. \& K. H. Pollock. 1983. Estimation methodology in contemporary small mammals capture-recapture studies. J. of Mamm., 64:253-260.

Orellana, R., I. Gerald \& C. Espadas. 2003. Presente pasado y futuro de los climas de la Península deYucatán. Pp. 37-51 In: Colunga G. \& A. Larqué S. (eds) Naturaleza y Sociedad en el Área Maya. Academia Mexicana de Ciencias. CYCY.

Ostfeld, R. H. 1985. Limiting resources and territoriality in microtine rodents. Amer. Nat. , 1:1-15.

Ramírez-Pulido, J., I. Lira, C. Müdespacher y A. Castro. 1989. Manejo y mantenimiento de colecciones mastozoológicas. Universidad Autónoma Metropolitana. 53-60 p.

Rasmussen-Terán, M. C. 2006. Fluctuación poblacional de Heteromys gaumeri, Allen y Chapman 1897 (Rodentia:Geomyidae) en una selva mediana subcaducifolia de Hobonil, Yucatán, México, durante el período de 1996-1998. Tesis de Licenciatura. FMVZ-UADY. Mérida, Yucatán, México. 64 p.

Romero, L. 1993. Biología de Lyomis pictus. Tesis Doctoral, Facultad de Ciencias UNAM. $107 \mathrm{pp}$.

Rudran, R. 1996. Method for marking mammals. Pp. 299-310. In: Measuring and monitoring biological diversity: Standard methods for mammals. D. E. Wilson, F. R. Cole, J. D. Nochols, R. Rudran y M. S. Foster (eds.). Smithsonian Institution Press. Washington, D. C. XXVII+409 pp.

Rzedowsky, J. 1987. Vegetación de México. Limusa. México. 431 pp.

Saenz, J. C. 1999. Movimientos y selección de micro-hábitat de una rata arborícola Ototylomys phyllotis (Rodentia: Muridae) en un bosque seco tropical. Brenesia, 52:61-64.

Sánchez-Cordero, V. 1993. Estudio poblacional de la rata espinosa Heteromys desmarestianus en una selva húmeda de Veracruz, México. Pp 301-316. In: Avances en el estudio 
de los mamíferos de México (R. A. Medellín y G. Ceballos, eds.). Asoc. Mex. Mast., A. C. México, D. F.

Santos-Moreno, A., M.a: Briones-Salas \& R. López Wilchis. 2007. Diferencias en algunos parámetros demográficos de Oryzomis chapmani (Rodentia: Muridae) asociadas a tres estados sucesionales del bosque mesófilo de montaña en Oaxaca, México. Acta Zool. Mex. (n.s). 23 (1): 123-137.

Stebbins, L. L. 1977. Energy requeriments during reproduction of Peromyscus maniculatus. Can. J. of Zool., 55:1701-1704.

Vazquez, L. B., R. A. Medellín \& G. N. Cameron. 2000. Pupulation and comunity ecology of small rodents in montane forest of western Mexico. J. of mamm., 81(1):77-85.

Wackerly, D.D., W. Mendenhall y R. L. Scheaffer. 2002. Estadística Matemática con Aplicaciones. $6^{\mathrm{a}}$ Ed. Thomson. México, D.F.

White, D. A. \& C. S. Hood. 2004. Vegetation patterns and environmental gradients in tropical dry forests of the northern Yucatan Peninsula. J. of Veg. Sci 15: 151-160.

Zalapa, S. S., M. H., F. A. Cervantes \& S. Guerrero. 2005. Ecología de Liomys pictus en tres áreas del bosque tropical subcaducifolio con diferente tiempo de regeneración en la costa noreste de Jalisco, México. Acta Zoo. Mex. (n.s). 21 (2): 1-14.

Zar, J. H. 1999. Bioestatistical Analisis. $4^{\text {a }}$ Ed. Prentice-Hall. New Jersey, USA. 863 p. 
